Pacific

Journal of

Mathematics

\title{
THE GENUS FILTRATION IN THE SMOOTH CONCORDANCE GROUP
}

\author{
SHIDA WANG
}




\title{
THE GENUS FILTRATION IN THE SMOOTH CONCORDANCE GROUP
}

\author{
SHIDA WANG
}

\begin{abstract}
We define a filtration of the smooth concordance group based on the genus of representative knots. We use the Heegaard Floer $\varepsilon$ - and $\Upsilon$-invariants to prove the quotient groups with respect to this filtration are infinitely generated. Results are applied to three infinite families of topologically slice knots.
\end{abstract}

\section{Introduction}

Let $\mathcal{C}$ be the smooth concordance group. Let $\mathcal{G}_{k}$ denote the subgroup of $\mathcal{C}$ generated by knots of genus not greater than $k$. Clearly $\mathcal{G}_{0} \subseteq \mathcal{G}_{1} \subseteq \mathcal{G}_{2} \subseteq \cdots \subseteq \mathcal{G}_{k} \subseteq \cdots \subseteq \mathcal{C}$ and $\bigcup_{k=1}^{\infty} \mathcal{G}_{k}=\mathcal{C}$. This gives a filtration of $\mathcal{C}$. We call it the genus filtration.

There is another way to understand $\mathcal{G}_{k}$. Recall that the concordance genus $g_{c}$ of a knot $K$ is defined to be the minimal genus of a knot $K^{\prime}$ concordant to $K$. It is obvious that $g_{c}(K)=\min \left\{k \mid K\right.$ is concordant to $K^{\prime}$ and $\left.g\left(K^{\prime}\right) \leq k\right\}$. This motivates the following definition.

Definition 1.1. The splitting concordance genus of a knot $K$ is $g_{\mathrm{sp}}(K):=\min \left\{k \mid K\right.$ is concordant to $K_{1} \# \ldots \# K_{m}$ for some $m$

$$
\text { and } \left.g\left(K_{1}\right), \ldots, g\left(K_{m}\right) \leq k\right\} \text {. }
$$

That is to say, $g_{\text {sp }}(K)$ is the filtration level of $K$ in $\mathcal{G}_{0} \subseteq \mathcal{G}_{1} \subseteq \cdots \subseteq \mathcal{G}_{k} \subseteq \cdots \subseteq \mathcal{C}$. By [Endo 1995], $\mathcal{G}_{1}$ contains a $\mathbb{Z}^{\infty}$ subgroup whose elements are topologically slice.

Let $\mathcal{C}_{T S} \subseteq \mathcal{C}$ be the subgroup of topologically slice knots. Recently several results have appeared which reveal that the group $\mathcal{C}_{T S}$ is quite large. For example, in [Hom 2015a; Ozsvath et al. 2014] it is shown that $\mathcal{C}_{T S}$ contains $\mathbb{Z}^{\infty}$ as a direct summand. In [Hedden et al. 2012] it is shown that $\mathcal{C}_{T S}$ contains $\mathbb{Z}^{\infty}$ as a subgroup whose nonzero elements are not concordant to knots of Alexander polynomial one. In [Hedden et al. 2016] it is shown that $\mathcal{C}_{T S}$ contains $\mathbb{Z}_{2}^{\infty}$ as a subgroup whose nonzero elements are not concordant to knots of Alexander polynomial one.

MSC2010: primary 57N70; secondary 57M25.

Keywords: knot concordance, genus, Heegaard Floer homology. 
We will show that $\mathcal{C}_{T S}$ is large in another sense. We will prove $\mathcal{C}_{T S} \nsubseteq \subseteq \mathcal{G}_{k}$ for any $k$. Moreover, the difference between $\mathcal{C}_{T S}$ and $\mathcal{G}_{k}$ is large. Corollary 1.4 states that $\mathcal{C}_{T S} /\left(\mathcal{C}_{T S} \cap \mathcal{G}_{k}\right)$ contains a direct summand isomorphic to $\mathbb{Z}^{\infty}$.

Our examples will be built from those of [Hom 2014a; Ozsvath et al. 2014]. Let $\mathrm{Wh}(K)$ denote the untwisted Whitehead double of a knot $K$. Additionally let $K_{p, q}$ denote the $(p, q)$-cable of $K$, let $J_{n}=\left(\mathrm{Wh}\left(T_{2,3}\right)\right)_{n, n+1} \#-T_{n, n+1}$, and let $J_{n}^{\prime}=\left(\mathrm{Wh}\left(T_{2,3}\right)\right)_{n, 2 n-1} \#-T_{n, 2 n-1}$. These knots are topologically slice and used to prove the following theorems.

Theorem 1 [Hom 2015a, Theorem 1]. The group $\mathcal{C}_{T S}$ contains a summand which is isomorphic to $\mathbb{Z}^{\infty}$ and generated by $\left\{J_{n}\right\}_{n=2}^{\infty}$.

Theorem 2 [Ozsvath et al. 2014, Theorem 1.20]. The topologically slice knots $\left\{J_{n}^{\prime}\right\}_{n=2}^{\infty}$ form a basis for a free direct summand of $\mathcal{C}_{T S}$.

We will prove the following results.

Theorem 1.2. $\left\{J_{n}\right\}_{n=k}^{\infty}$ forms a basis for a $\mathbb{Z}^{\infty}$ summand of $\mathcal{C}_{T S} /\left(\mathcal{C}_{T S} \cap \mathcal{G}_{\lfloor k / 2\rfloor}\right)$ for any $k \geq 2$.

Theorem 1.3. $\left\{J_{n}^{\prime}\right\}_{n=k}^{\infty}$ forms a basis for a $\mathbb{Z}^{\infty}$ summand of $\mathcal{C}_{T S} /\left(\mathcal{C}_{T S} \cap \mathcal{G}_{k-1}\right)$ for any $k \geq 2$.

Hence we have the following consequence.

Corollary 1.4. For any $k \in \mathbb{N}$ we have $\mathcal{C}_{T S} \nsubseteq \mathcal{G}_{k}$. Moreover, the quotient group $\mathcal{C}_{T S} /\left(\mathcal{C}_{T S} \cap \mathcal{G}_{k}\right)$ contains a direct summand isomorphic to $\mathbb{Z}^{\infty}$.

One can define another subgroup $\mathcal{H}_{k}$ of $\mathcal{C}$ generated by knots of 4-genus not greater than $k$. Clearly $\mathcal{G}_{k} \subseteq \mathcal{H}_{k}$. It is natural to ask whether $\mathcal{H}_{k} / \mathcal{G}_{k}$ is infinitely generated. We show the answer is affirmative by proving the following:

Theorem 1.5. The quotient group $\mathcal{C}_{T S} /\left(\mathcal{C}_{T S} \cap \mathcal{G}_{k}\right)$ contains a subgroup isomorphic to $\mathbb{Z}^{\infty}$ whose basis elements have slice genus 1 for any $k \geq 2$.

Conjecture 1.6. (1) For any $k \in \mathbb{N}$, the quotient $\left(\mathcal{C}_{T S} \cap \mathcal{G}_{k+1}\right) /\left(\mathcal{C}_{T S} \cap \mathcal{G}_{k}\right)$ contains a direct summand isomorphic to $\mathbb{Z}^{\infty}$ whose basis elements have slice genus 1 .

(2) For any $k \in \mathbb{N}$, the group $\mathcal{C} / \mathcal{H}_{n}$ is nontrivial.

This paper is organized as follows. In Section 2 we use Alexander polynomials to prove the splitting concordance genus can be arbitrarily large. In Section 3 we review Hom's $\varepsilon$-invariant and develop an obstruction, which is used to prove Theorems 1.2 and 1.5. In Section 4 we use the $\Upsilon$-invariant to develop an obstruction and prove Theorem 1.3. 


\section{A first glance at the genus filtration}

Given a knot $K$, let $\Delta_{K}(t)$ be its Alexander polynomial, and breadth $\left(\Delta_{K}(t)\right)$ be the maximal exponent of $\Delta_{K}(t)$ minus the minimal exponent of $\Delta_{K}(t)$. Recall that for any $K$, breadth $\left(\Delta_{K}(t)\right) \leq 2 g(K)$. Moreover, if $K$ is slice, recall that it must satisfy the Fox-Milnor condition, factoring as $t^{ \pm n} f(t) f\left(t^{-1}\right)$. Based on these facts, we can prove the following theorem, generalizing [Livingston 2004, Theorem 2.2].

Proposition 2.1. For any knot $K$, if $p(t)$ appears an odd number of times in the irreducible factorization of $\Delta_{K}(t)$ in $\mathbb{Z}\left[t, t^{-1}\right]$, then

$$
g_{\mathrm{sp}}(K) \geq \frac{1}{2} \operatorname{breadth}(p(t)) .
$$

Proof. By definition, we can choose knots $K_{1}, \ldots, K_{m}$ such that $K$ is concordant to $K_{1} \# \cdots \# K_{m}$ and $g\left(K_{i}\right) \leq g_{\text {sp }}(K)$ for each $1 \leq i \leq m$. Thus $K \#-K_{1} \# \cdots \#-K_{m}$ is a slice knot and its Alexander polynomial $\Delta_{K}(t) \Delta_{K_{1}}(t) \cdots \Delta_{K_{m}}(t)$ must factor as $t^{ \pm n} f(t) f\left(t^{-1}\right)$ for some $f \in \mathbb{Z}\left[t, t^{-1}\right]$. If some $p(t)$ appears an odd number of times in the irreducible factorization of $\Delta_{K}(t)$, it must appear in the irreducible factorization of one of $\Delta_{K_{1}}(t), \ldots, \Delta_{K_{m}}(t)$. Since $2 g_{\mathrm{sp}}(K) \geq \operatorname{breadth}\left(\Delta_{K_{i}}(t)\right)$ for each $1 \leq i \leq m$, we conclude that $2 g_{\mathrm{sp}}(K) \geq \operatorname{breadth}(p(t))$.

Example 2.2. The Alexander polynomial of the torus knot $T_{p, q}$ is

$$
\Delta_{T_{p, q}}(t)=\left(\left(t^{p q}-1\right)(t-1)\right) /\left(\left(t^{p}-1\right)\left(t^{q}-1\right)\right),
$$

in whose irreducible factorization the cyclotomic polynomial $\Phi_{p q}$ appears exactly once. Hence $g_{\mathrm{sp}}\left(T_{p, q}\right) \geq \varphi(p q) / 2$, where $\varphi$ is Euler's totient function. If $p$ and $q$ are prime, we have $g_{\mathrm{sp}}\left(T_{p, q}\right) \geq((p-1)(q-1)) / 2$. This is actually an equality, because $g\left(T_{p, q}\right)=((p-1)(q-1)) / 2$.

Corollary 2.3. $\mathcal{C} / \mathcal{G}_{k}$ is nontrivial for any $k \in \mathbb{N}$.

Working a little harder, we can show the following.

Proposition 2.4. $\mathcal{C} / \mathcal{G}_{k}$ contains an infinitely generated free subgroup for any $k \in \mathbb{N}$.

Proof. Let $\left\{p_{n}\right\}_{n=1}^{\infty}$ be a sequence of strictly increasing prime numbers with $p_{1}>k$. We will prove that the torus knots $\left\{T_{p_{2 n-1}, p_{2 n}}\right\}_{n=1}^{\infty}$ are linearly independent in $\mathcal{C} / \mathcal{G}_{k}$.

Suppose towards a contradiction that $\#_{i=1}^{l} c_{i} T_{p_{2 n_{i}-1}, p_{2 n_{i}}}$, where $0<n_{1}<\cdots<n_{l}$ and $c_{1}, \ldots, c_{l}$ are nonzero integers, is concordant to $K_{1} \# \ldots \# K_{m}$ with $g\left(K_{j}\right) \leq k$ for $1 \leq j \leq m$. Notice that $\Delta_{T_{p_{2 n_{i}-1}, p_{2 n_{i}}}}(t)=\Phi_{p_{2 n_{i}-1} p_{2 n_{i}}}$, where $\Phi_{p_{2 n_{i}-1} p_{2 n_{i}}}$ is the cyclotomic polynomial, which is irreducible of degree $\left(p_{2 n_{i}-1}-1\right)\left(p_{2 n_{i}}-1\right)$. By a combinatorial formula [Litherland 1979, Proposition 1] for the TristramLevine signature functions of torus knots, $\sigma_{\omega}\left(T_{p_{2 n_{i}-1}, p_{2 n_{i}}}\right)$ jumps by \pm 2 at the primitive $\left(p_{2 n_{i}-1} p_{2 n_{i}}\right)$-th roots of unity. Since the products $p_{2 n_{i}-1} p_{2 n_{i}}$ are distinct for $i=1, \ldots, l$, we know $\sigma_{\omega}\left(\#_{i=1}^{l} c_{i} T_{p_{2 n_{i}-1}, p_{2 n_{i}}}\right)$ has a jump discontinuity 
at a primitive $\left(p_{2 n_{1}-1} p_{2 n_{1}}\right)$-th root of unity. Hence $\sigma_{\omega}\left(K_{1} \# \cdots \# K_{m}\right)$ also has a jump discontinuity at a primitive $\left(p_{2 n_{1}-1} p_{2 n_{1}}\right)$-th root of unity, and so does one of $\sigma_{\omega}\left(K_{1}\right), \ldots, \sigma_{\omega}\left(K_{m}\right)$. Without loss of generality, assume that $\sigma_{\omega}\left(K_{1}\right)$ has a jump discontinuity at a primitive $\left(p_{2 n_{1}-1} p_{2 n_{1}}\right)$-th root of unity. Since jump discontinuities of the Tristram-Levine signature function can only appear at roots of the Alexander polynomial, it follows that $\Delta_{K_{1}}(t)$ has a root at a primitive $\left(p_{2 n_{1}-1} p_{2 n_{1}}\right)$-th root of unity and thus is divisible by $\Phi_{p_{2 n_{i}-1} p_{2 n_{i}}}$, but this is impossible because

$$
\operatorname{deg} \Delta_{K_{1}}(t) \leq 2 g\left(K_{1}\right) \leq 2 k<\left(p_{2 n_{i}-1}-1\right)\left(p_{2 n_{i}}-1\right) .
$$

\section{Obstruction by $\varepsilon$-invariant}

We assume the reader is familiar with knot Floer homology defined by Ozsváth and Szabó [2004b] and independently Rasmussen [2003] and the $\varepsilon$-invariant defined by Hom [2014a]. We briefly recall some of their properties for later use.

The knot Floer complex and $\varepsilon$-invariant. The knot Floer complex associates to a knot $K \subset S^{3}$ a doubly filtered, free, finitely generated chain complex over $\mathbb{F}\left[U, U^{-1}\right]$, denoted by $C F K^{\infty}(K)$, where $\mathbb{F}$ is the field with two elements. The two filtrations are called the algebraic and Alexander filtrations and the grading of the chain complex is called the homological or Maslov grading. Multiplication by $U$ shifts each filtration down by one and lowers the homological grading by two. $C F K^{\infty}(K)$ is an invariant of $K$ up to filtered chain homotopy equivalence. Furthermore, up to filtered chain homotopy equivalence, one can assume the differential strictly lowers at least one of the filtrations [Rasmussen 2003].

A quick corollary from [Ozsváth and Szabó 2004a, Theorem 1.2] is the following.

Proposition 3.1. If $K$ has genus $g$, then there exists a representative of the filtered chain homotopy equivalence class of $\mathrm{CFK}^{\infty}(K)$ all of whose elements have filtration levels $(i, j)$ such that $-g \leq i-j \leq g$.

For a subset $S \subseteq \mathbb{Z} \oplus \mathbb{Z}$ that is downward closed under the standard product partial order on $\mathbb{Z} \oplus \mathbb{Z}$, let $C\{S\}$ denote the subcomplex of $C F K^{\infty}(K)$ generated by elements with filtration levels in $S$. If $S$ is the difference of two such subsets, let $C\{S\}$ denote the corresponding subquotient complex of $C F K^{\infty}(K)$. For example, $C\{i=0\}=C\{i \leq 0\} / C\{i<0\}=C F K^{\infty}(K)\{i \leq 0\} / C F K^{\infty}(K)\{i<0\}$. The invariant $\tau(K)=\min \{s \mid$ the inclusion map $C\{i=0, j \leq s\} \rightarrow C\{i=0\}$

\section{induces a nontrivial map on homology\}}

is proven to be a smooth concordance invariant in [Ozsváth and Szabó 2003].

For any knot $K$, Hom [2014a] defines an invariant called $\varepsilon$ taking on values -1 , 0 or 1 , which has the following properties. 
Proposition 3.2 [Hom 2014a, Proposition 3.6]. The invariant $\varepsilon$ satisfies the following properties:

(1) If $K$ is smoothly slice, then $\varepsilon(K)=0$.

(2) $\varepsilon(-K)=-\varepsilon(K)$.

(3) If $\varepsilon(K)=\varepsilon\left(K^{\prime}\right)$, then $\varepsilon\left(K \# K^{\prime}\right)=\varepsilon(K)=\varepsilon\left(K^{\prime}\right)$.

(4) If $\varepsilon(K)=0$, then $\varepsilon\left(K \# K^{\prime}\right)=\varepsilon\left(K^{\prime}\right)$.

Thus the relation $\sim$, defined by $K \sim K^{\prime} \Leftrightarrow \varepsilon\left(K \#-K^{\prime}\right)=0$, is an equivalence relation coarser than smooth concordance. It gives an equivalence relation on $\mathcal{C}$ called $\varepsilon$-equivalence. The $\varepsilon$-equivalence class of $K$ is denoted by $\llbracket K \rrbracket$. The set of all $\varepsilon$-equivalence classes forms a group $\mathcal{F}$ (also denoted by $\mathcal{C} \mathcal{F} \mathcal{K}$ in [Hom 2015a]), which is a quotient group of $\mathcal{C}$. The kernel of the natural homomorphism from $\mathcal{C}$ to $\mathcal{F}$ is $\{[K] \in \mathcal{C} \mid \varepsilon(K)=0\}$, where $[K]$ denotes the concordance class of $K$.

According to [Hom 2014b, Proposition 4.1], $\varepsilon$ induces a total order on $\mathcal{F}$. The proof uses Proposition 3.2. The total order is defined by

$$
\llbracket K \rrbracket>\llbracket K^{\prime} \rrbracket \Leftrightarrow \varepsilon\left(K \#-K^{\prime}\right)=1 .
$$

Moreover, this order respects the addition operation on $\mathcal{F}$. Therefore there is a quotient homomorphism from $\mathcal{C}$ to the totally ordered abelian group $\mathcal{F}$, which can be used to show linear independence in $\mathcal{C}$.

Some facts about totally ordered abelian groups. Let $G$ be a totally ordered abelian group, that is an abelian group with a total order respecting the addition operation. Denote its identity element by 0 .

The absolute value of an element $a \in G$ is defined to be

$$
|a|=\left\{\begin{aligned}
a & \text { if } a \geq 0 \\
-a & \text { if } a<0 .
\end{aligned}\right.
$$

Definition 3.3. Two nonzero elements $a$ and $b$ of $G$ are Archimedean equivalent, denoted by $a \sim_{A} b$, if there exists a natural number $N$ such that $N \cdot|a|>|b|$ and $N \cdot|b|>|a|$. If $a$ and $b$ are not Archimedean equivalent and $|a|<|b|$, we say that $b$ dominates $a$. We write $a \ll b$ if $a>0, b>0$ and $b$ dominates $a$.

Property A. An element $a \in G$ satisfies Property $A$ if for every $b \in G$ such that $b \sim_{A} a$, we have that $b=k a+c$, where $k$ is an integer and $c$ is dominated by $a$.

We have the following two facts:

Lemma 3.4 [Hom 2014b, Lemma 4.7]. If $0<a_{1} \ll a_{2} \ll a_{3} \ll \cdots$ in $G$, then $a_{1}, a_{2}, a_{3}, \ldots$ are linearly independent in $G$. 
Lemma 3.5 [Hom 2015a, Proposition 1.3]. If $0<a_{1} \ll a_{2} \ll a_{3} \ll \cdots$ in $G$ and each $a_{i}$ satisfies Property $A$, then $a_{1}, a_{2}, a_{3}, \ldots$ generate (as a basis) a direct summand isomorphic to $\mathbb{Z}^{\infty}$ in $G$.

The following lemmas are proven in [Hom 2014b] and [Hom 2015a] respectively.

Lemma 3.6 [Hom 2014b, Remark 4.9]. We have $0<\llbracket J_{n} \rrbracket \ll \llbracket J_{n+1} \rrbracket$ for any $n \geq 2$.

Lemma 3.7 [Hom 2015a, Proposition 4.1, Lemmas 5.2 and 5.3]. The class $\llbracket J_{n} \rrbracket$ satisfies Property A for any $n \geq 2$.

It is straightforward to check that $\{a:|a| \ll x\}$ is a subgroup of $G$ for any $x>0$ in $G$. Denote this subgroup by $G_{x}$. Let $\varphi_{x}$ be the quotient homomorphism. Define a relation $<$ in $G / G_{x}$ by $\varphi_{x}(a)<\varphi_{x}(b)$ if and only if $a<b$ and $b-a \notin G_{x}$.

Proposition 3.8. The relation $<$ makes $G / G_{x}$ into a totally ordered abelian group with the following properties: If $0<a \ll b$ in $G$ and $b \notin G_{x}$, then $0 \leq \varphi_{x}(a) \ll \varphi_{x}(b)$ in $G / G_{x}$. If a satisfies Property $A$ in $G$, then $\varphi_{x}(a)$ satisfies Property $A$ in $G / G_{x}$.

Proof. First we check that the relation $<$ in $G / G_{x}$ is well defined. Suppose $\varphi_{x}(a)<\varphi_{x}(b)$. Let $c \in G_{x}$. We must show $\varphi_{x}(a+c)<\varphi_{x}(b)$ and $\varphi_{x}(a)<\varphi_{x}(b+c)$. Since $b-a>0$ and $b-a \notin G_{x}$ it is easy to verify that $b-a \gg|y|$ for any $y \in G_{x}$. Thus $b-a \pm c>0$. Additionally $b-a \notin G_{x}$ implies $b-a \pm c \notin G_{x}$. Hence $\varphi_{x}(a+c)<\varphi_{x}(b)$ and $\varphi_{x}(a)<\varphi_{x}(b+c)$, which means the definition does not depend on the choices of $a$ and $b$.

Next we verify $<$ is a strict total order on $G / G_{x}$ that respects the addition operation. For trichotomy, let $\varphi_{x}(a)$ and $\varphi_{x}(b)$ be two distinct elements in $G / G_{x}$. Then $b-a \notin G_{x}$. Thus $b-a \neq 0$ and exactly one of $a<b$ and $b<a$ is true. Hence exactly one of $\varphi_{x}(a)<\varphi_{x}(b)$ and $\varphi_{x}(b)<\varphi_{x}(a)$ is true by definition. For transitivity, let $\varphi_{x}(a), \varphi_{x}(b), \varphi_{x}(c) \in G / G_{x}$ satisfy $\varphi_{x}(a)<\varphi_{x}(b)$ and $\varphi_{x}(b)<\varphi_{x}(c)$. Then $a<b, b<c$ and $b-a, c-b \notin G_{x}$. Immediately $a<c$. Suppose towards a contradiction that $c-a \in G_{x}$. Then the fact that $b-a \gg|y|$ for any $y \in G_{x}$ implies $b-a-(c-a)>0$, which contradicts $b<c$. Hence $c-a \notin G_{x}$ and $\varphi_{x}(a)<\varphi_{x}(c)$ by definition. For consistency with the addition operation, let $\varphi_{x}(a)$, $\varphi_{x}(b), \varphi_{x}(c) \in G / G_{x}$ and $\varphi_{x}(a)<\varphi_{x}(b)$. Then $a<b$ and $b-a \notin G_{x}$. Thus $a+c<b+c$ and $(b+c)-(a+c) \notin G_{x}$. Hence $\varphi_{x}(a)+\varphi_{x}(c)=\varphi_{x}(a+c)<$ $\varphi_{x}(b+c)=\varphi_{x}(b)+\varphi_{x}(c)$ by definition.

Next, we show that if $b$ dominates $a$ in $G$ and $b \notin G_{x}$, then $\varphi_{x}(b)$ dominates $\varphi_{x}(a)$. Suppose $0<a \ll b$ in $G$ and $b \notin G_{x}$. Then $0<N a<b$ for any $N \in \mathbb{N}$. Additionally, the fact that $b \gg|y|$ for any $y \in G_{x}$ implies $N a+y<b, \forall y \in G_{x}$. It follows that $b-N a>0$ and that $b-N a \notin G_{x}$. Hence $0 \leq \varphi_{x}(a) \ll \varphi_{x}(b)$ in $G / G_{x}$ by definition.

Finally we show that if $a$ has Property A in $G$, then $\varphi_{x}(a)$ has Property A in $G / G_{x}$. Suppose $a$ satisfies Property A in $G$, that is, if $b \sim_{A} a$ in $G$ then $b=k a+c$ for some integer $k$ and some $c \in G$ dominated by $a$. Without loss of generality 
we assume $\varphi_{x}(a) \neq 0$. Let $\varphi_{x}(b) \sim_{A} \varphi_{x}(a)$, so $b \sim_{A} a$ in $G$. Otherwise either $|a| \ll|b|$ or $|b| \ll|a|$, which would imply $\left|\varphi_{x}(a)\right| \ll\left|\varphi_{x}(b)\right|$ or $\left|\varphi_{x}(b)\right| \ll\left|\varphi_{x}(a)\right|$. Thus $b=k a+c$ for some integer $k$ and some $c \in G$ dominated by $a$. Thus $\varphi_{x}(b)=k \varphi_{x}(a)+\varphi_{x}(c)$. Since $c$ is dominated by $a$, we know $\varphi_{x}(c)$ is dominated by $\varphi_{x}(a)$. Hence $\varphi_{x}(a)$ satisfies Property A in $G / G_{x}$.

Restriction on the Archimedean equivalence class by genus. Given a knot $K$ with $\varepsilon(K)=1$, Hom [2015a, Section 3] defines a tuple of numerical invariants $\boldsymbol{a}^{+}(K)=\left(a_{1}(K), \ldots, a_{n}(K)\right)$. Here each $a_{i}(K)$ is a positive integer, and the number $n$ depends on $K$. It is shown that $\boldsymbol{a}^{+}(K)$ is an invariant of the $\varepsilon$-equivalence class $\llbracket K \rrbracket$ (see [Hom 2015a, Proposition 3.1]).

Computations in [Hom 2014b] show the following result.

Lemma 3.9 [Hom 2014b, p.568]. We have $\boldsymbol{a}^{+}\left(J_{p}\right)=(1, p, \ldots)$.

The integers $a_{1}$ and $a_{2}$ are useful in determining domination.

Lemma 3.10 [Hom 2014b, Lemmas 6.3 and 6.4]. If $\boldsymbol{a}^{+}(K)=\left(a_{1}(K), \ldots\right)$ and $\boldsymbol{a}^{+}\left(K^{\prime}\right)=\left(a_{1}\left(K^{\prime}\right), \ldots\right)$ with $a_{1}(K)>a_{1}\left(K^{\prime}\right)>0$, then $\llbracket K \rrbracket \ll \llbracket K^{\prime} \rrbracket$.

Additionally, if $\boldsymbol{a}^{+}(K)=\left(a_{1}(K), a_{2}(K), \ldots\right)$ and $\boldsymbol{a}^{+}\left(K^{\prime}\right)=\left(a_{1}\left(K^{\prime}\right), a_{2}\left(K^{\prime}\right), \ldots\right)$ with $a_{1}(K)=a_{1}\left(K^{\prime}\right)>0$ and $a_{2}(K)>a_{2}\left(K^{\prime}\right)>0$, then $\llbracket K \rrbracket \gg \llbracket K^{\prime} \rrbracket$.

Based on Proposition 3.1, the following is shown.

Lemma 3.11 ([Hom 2015b, Theorem 1.2 and Lemma 2.3]). Suppose that $\varepsilon(K)=1$, and $a_{2}(K)$ is defined, then $\left|\tau(K)-a_{1}(K)-a_{2}(K)\right| \leq g(K)$.

Next we prove our obstruction theorem.

Proposition 3.12. Suppose $J$ is a knot with $\boldsymbol{a}^{+}(J)=(1, b, \ldots)$ with $b \geq 2 n$ for some positive integer $n$. Then for any knot $K \in \mathcal{G}_{n}$, we have $|\llbracket K \rrbracket| \ll \llbracket J \rrbracket$.

Proof. Before proving the proposition for $K \in \mathcal{G}_{n}$, first consider the case $g(K) \leq n$. We may further assume that $\llbracket K \rrbracket>0$, since $\llbracket-K \rrbracket>0$ if $\llbracket K \rrbracket<0$ and the proposition is trivial if $\llbracket K \rrbracket=0$. Notice that $a_{1}(K)$ is always defined [Hom 2014b, $\S 6]$. If $a_{1}(K)>1$, then $\llbracket K \rrbracket \ll \llbracket J \rrbracket$ by Lemma 3.10. If $a_{1}(K)=1$, then $a_{2}(K)$ is defined [Hom 2015a, Lemma 3.7]. Observe that $\tau(K)-a_{1}(K)-a_{2}(K) \geq-g(K)$ by Lemma 3.11. Combining this with $\tau(K) \leq g_{4}(K) \leq g(K)$, it follows that $g(K)-a_{1}(K)-a_{2}(K) \geq-g(K)$. This implies $a_{2}(K) \leq 2 n-1$, if $a_{1}(K)=1$. Hence $|\llbracket K \rrbracket| \ll \llbracket J \rrbracket$ by Lemma 3.10.

Generally, let $K \in \mathcal{G}_{n}$. Then $K=K_{1}+\cdots+K_{m}$, where $g\left(K_{i}\right) \leq n$ for $i=1, \ldots, m$. Since $\llbracket K \rrbracket=\llbracket K_{1} \rrbracket+\cdots+\llbracket K_{m} \rrbracket$, we know $|\llbracket K \rrbracket| \leq\left|\llbracket K_{1} \rrbracket\right|+\cdots+\left|\llbracket K_{m} \rrbracket\right|$. Then the conclusion follows from the last paragraph. 


\section{Applying the obstruction to concrete families of knots.}

Proof of Theorem 1.2. Fix an integer $k \geq 2$. Under the quotient homomorphism from $\mathcal{C}$ to $\mathcal{F}$, the image of $\mathcal{G}_{\lfloor k / 2\rfloor}$ is included in $\mathcal{F}_{\llbracket J_{k} \rrbracket}=\left\{\llbracket K \rrbracket:|\llbracket K \rrbracket| \ll \llbracket J_{k} \rrbracket\right\}$ by Proposition 3.12 and Lemma 3.9. This gives a homomorphism from $\mathcal{C} / \mathcal{G}\lfloor k / 2\rfloor$ to $\mathcal{F} / \mathcal{F}_{\llbracket J_{k} \rrbracket}$. By Lemma 3.6 and Proposition 3.8, the family $\left\{J_{n}\right\}_{n=k}^{\infty}$ maps to a family of elements with Property A and each term is dominated by the next. Hence $\left\{J_{n}\right\}_{n=k}^{\infty}$ forms a basis of a direct summand isomorphic to $\mathbb{Z}^{\infty}$ by Lemma 3.5. Note that since the $J_{n}$ are topologically slice, the above argument can be restricted to the subgroup $\mathcal{C}_{T S} /\left(\mathcal{C}_{T S} \cap \mathcal{G}_{\lfloor k / 2\rfloor}\right)$ of $\mathcal{C} / \mathcal{G}_{\lfloor k / 2\rfloor}$ to complete the proof.

Proof of Theorem 1.5. Instead of $\left\{J_{n}\right\}$, we use another family of topologically slice knots $\left\{L_{n}\right\}$, where $L_{n}=\left(\mathrm{Wh}\left(T_{2,3}\right)\right)_{n, 1} \#-\left(\mathrm{Wh}\left(T_{2,3}\right)\right)_{n-1,1}$. These knots have slice genus 1 [Hom 2015b, Lemma 3.1]. Additionally, Hom [2015b] computes that $a_{1}\left(L_{n}\right)=1$ and $a_{2}\left(L_{n}\right)=n$. By the same argument as the above proof, except for applying Lemma 3.4 rather than Lemma 3.5, we immediately know $\left\{L_{n}\right\}_{n=2 k}^{\infty}$ are linearly independent in $\mathcal{C}_{T S} /\left(\mathcal{C}_{T S} \cap \mathcal{G}_{k}\right)$.

\section{Obstruction by $\Upsilon$-invariant}

Ozsvath et al. [2014] introduced a new family of knot invariants, $\Upsilon_{K}(t)$. We refer the reader to their construction, and confine ourselves to recalling the basic properties of the $\Upsilon$-invariant.

For any knot $K$, the invariant $\Upsilon_{K}(t)$ is a piecewise linear function on [0,2] whose derivative has finitely many discontinuities [Ozsvath et al. 2014, Proposition 1.4]. Thus, one can define $\Delta \Upsilon_{K}^{\prime}\left(t_{0}\right)=\lim _{t \rightarrow t_{0}^{+}} \Upsilon_{K}^{\prime}(t)-\lim _{t \rightarrow t_{0}^{-}} \Upsilon_{K}^{\prime}(t)$ for any $t_{0} \in(0,2)$.

As an example, the authors of [Ozsvath et al. 2014] compute the family $\left\{J_{n}^{\prime}\right\}$ :

$$
\Delta \Upsilon_{J_{n}^{\prime}}^{\prime}(t)= \begin{cases}0 & \text { for } t<2 /(2 n-1), \\ 2 n-1 & \text { for } t=2 /(2 n-1) .\end{cases}
$$

In [Ozsvath et al. 2014, Corollary 1.12] it is shown that $\Upsilon$ gives a homomorphism from $\mathcal{C}$ to the vector space of continuous functions on [0,2]. Additionally,

$$
K \mapsto \begin{cases}(1 / q) \Delta \Upsilon_{K}^{\prime}(p / q) & \text { if } p \text { is even, } \\ (1 / 2 q) \Delta \Upsilon_{K}^{\prime}(p / q) & \text { if } p \text { is odd, }\end{cases}
$$

gives a homomorphism from $\mathcal{C}$ to $\mathbb{Z}$ for any $p / q \in(0,2) \cap \mathbb{Q}$.

The location of singularities of $\Upsilon$ is related to the genus of the knot, as in the following proposition. The proof of this proposition, much like that of Lemma 3.11, is based on the fact in Proposition 3.1.

Proposition 4.1 [Livingston 2015, Theorem 8.2]. Suppose that $\Delta \Upsilon_{K}^{\prime}(t)$ is nonzero at $t=p / q$ with $\operatorname{gcd}(p, q)=1$. Then $q \leq g(K)$ if $p$ is odd, and $q \leq 2 g(K)$ if $p$ is even. 
With this proposition, we can easily prove our obstruction theorem.

Proposition 4.2. Suppose $K \in \mathcal{G}_{n}$ for some positive integer $n$. Then $\Delta \Upsilon_{K}^{\prime}(t)=0$ for $t \in(0,1 / n) \cap \mathbb{Q}$.

Proof. Before proving the proposition for $K \in \mathcal{G}_{n}$, first consider the case $g(K) \leq n$. If $\Upsilon_{K}(t)$ has a singularity at a rational number $p / q$ with $\operatorname{gcd}(p, q)=1$, then Proposition 4.1 implies $p / q \geq 1 / n$.

Generally, let $K \in \mathcal{G}_{n}$. Then $K=K_{1}+\cdots+K_{m}$, where $g\left(K_{i}\right) \leq n$ for $i=1, \ldots, m$. If $\Upsilon_{K}(t)$ has a singularity at a rational number $p / q$, then so does one of $\Upsilon_{K_{1}}(t), \ldots, \Upsilon_{K_{m}}(t)$, since $\Upsilon$ is a homomorphism. The conclusion follows from the last paragraph.

Proof of Theorem 1.3. Fix an integer $k \geq 2$. If $K \in \mathcal{G}_{k-1}$, then $\Upsilon_{K}(t)$ has no singularities on $(0,1 /(k-1)) \cap \mathbb{Q}$. Thus $\left\{K \mapsto 1 /(2 n-1) \Delta \Upsilon_{K}^{\prime}(2 /(2 n-1))\right\}_{n=k}^{\infty}$ gives a homomorphism from $\mathcal{C} / \mathcal{G}_{k-1}$ to $\mathbb{Z}^{\infty}$. Hence $\left\{J_{n}^{\prime}\right\}_{n=k}^{\infty}$ form a basis for a $\mathbb{Z}^{\infty}$ summand of $\mathcal{C} / \mathcal{G}_{k-1}$. Note that since the $J_{n}^{\prime}$ are topologically slice, the above argument can be restricted to the $\operatorname{subgroup} \mathcal{C}_{T S} /\left(\mathcal{C}_{T S} \cap \mathcal{G}_{k-1}\right)$ of $\mathcal{C} / \mathcal{G}_{k-1}$ to complete the proof.

\section{Acknowledgements}

The author wishes to express sincere thanks to Professor Charles Livingston for proposing this study and carefully reading a draft of this paper. Thanks also to Jennifer Hom for valuable comments on a draft and to the referee for very detailed suggestions on writing style and grammar.

\section{References}

[Endo 1995] H. Endo, "Linear independence of topologically slice knots in the smooth cobordism group", Topology Appl. 63:3 (1995), 257-262. MR 1334309

[Hedden et al. 2012] M. Hedden, C. Livingston, and D. Ruberman, "Topologically slice knots with nontrivial Alexander polynomial”, Adv. Math. 231:2 (2012), 913-939. MR 2955197

[Hedden et al. 2016] M. Hedden, S.-G. Kim, and C. Livingston, "Topologically slice knots of smooth concordance order two", J. Differential Geom. 102:3 (2016), 353-393. MR 3466802

[Hom 2014a] J. Hom, "Bordered Heegaard Floer homology and the tau-invariant of cable knots", J. Topol. 7:2 (2014), 287-326. MR 3217622

[Hom 2014b] J. Hom, "The knot Floer complex and the smooth concordance group", Comment. Math. Helv. 89:3 (2014), 537-570. MR 3260841

[Hom 2015a] J. Hom, "An infinite-rank summand of topologically slice knots", Geom. Topol. 19:2 (2015), 1063-1110. MR 3336278

[Hom 2015b] J. Hom, "On the concordance genus of topologically slice knots", Int. Math. Res. Not. 2015:5 (2015), 1295-1314. MR 3340357

[Litherland 1979] R. A. Litherland, "Signatures of iterated torus knots", pp. 71-84 in Topology of low-dimensional manifolds (Proc. Second Sussex Conf., Chelwood Gate, 1977), edited by R. A. Fenn, Lecture Notes in Math. 722, Springer, Berlin, 1979. MR 547456 
[Livingston 2004] C. Livingston, "The concordance genus of knots", Algebr. Geom. Topol. 4 (2004), 1-22. MR 2031909

[Livingston 2015] C. Livingston, "Notes on the knot concordance invariant Upsilon", preprint, 2015. arXiv $1412.0254 \mathrm{v} 2$

[Ozsváth and Szabó 2003] P. Ozsváth and Z. Szabó, "Knot Floer homology and the four-ball genus", Geom. Topol. 7 (2003), 615-639. MR 2026543

[Ozsváth and Szabó 2004a] P. Ozsváth and Z. Szabó, "Holomorphic disks and genus bounds", Geom. Topol. 8 (2004), 311-334. MR 2023281

[Ozsváth and Szabó 2004b] P. Ozsváth and Z. Szabó, "Holomorphic disks and knot invariants", Adv. Math. 186:1 (2004), 58-116. MR 2065507

[Ozsvath et al. 2014] P. Ozsvath, A. Stipsicz, and Z. Szabo, "Concordance homomorphisms from knot Floer homology", preprint, 2014. arXiv 1407.1795v2

[Rasmussen 2003] J. A. Rasmussen, Floer homology and knot complements, Ph.D. Thesis, Harvard University, 2003, Available at http://search.proquest.com/docview/305332635. MR 2704683

Received October 26, 2015. Revised June 23, 2016.

SHIDA WANG

DEPARTMENT OF MATHEMATICS

INDIANA UNIVERSITY

BLOOMINGTON, IN 47405

UNITED STATES

shidawang@gmail.com 


\title{
PACIFIC JOURNAL OF MATHEMATICS
}

Founded in 1951 by E. F. Beckenbach (1906-1982) and F. Wolf (1904-1989)

$$
\text { msp.org/pjm }
$$

\section{EDITORS}

\author{
Don Blasius (Managing Editor) \\ Department of Mathematics \\ University of California \\ Los Angeles, CA 90095-1555 \\ blasius@math.ucla.edu
}

\author{
Paul Balmer \\ Department of Mathematics \\ University of California \\ Los Angeles, CA 90095-1555 \\ balmer@math.ucla.edu \\ Robert Finn \\ Department of Mathematics \\ Stanford University \\ Stanford, CA 94305-2125 \\ finn@math.stanford.edu \\ Sorin Popa \\ Department of Mathematics \\ University of California \\ Los Angeles, CA 90095-1555 \\ popa@math.ucla.edu
}

\author{
Vyjayanthi Chari \\ Department of Mathematics \\ University of California \\ Riverside, CA 92521-0135 \\ chari@math.ucr.edu \\ Kefeng Liu \\ Department of Mathematics \\ University of California \\ Los Angeles, CA 90095-1555 \\ liu@math.ucla.edu \\ Igor Pak \\ Department of Mathematics \\ University of California \\ Los Angeles, CA 90095-1555 \\ pak.pjm@gmail.com \\ Paul Yang \\ Department of Mathematics \\ Princeton University \\ Princeton NJ 08544-1000 \\ yang@math.princeton.edu
}

\section{PRODUCTION}

Silvio Levy, Scientific Editor, production@msp.org

\section{SUPPORTING INSTITUTIONS}

ACADEMIA SINICA, TAIPEI

CALIFORNIA INST. OF TECHNOLOGY

STANFORD UNIVERSITY

UNIV. OF BRITISH COLUMBIA

UNIV. OF CALIFORNIA, BERKELEY

UNIV. OF CALIFORNIA, DAVIS

UNIV. OF CALIFORNIA, LOS ANGELES

UNIV. OF CALIFORNIA, RIVERSIDE

UNIV. OF CALIFORNIA, SAN DIEGO

UNIV. OF CALIF., SANTA BARBARA
KEIO UNIVERSITY

MATH. SCIENCES RESEARCH INSTITUTE

NEW MEXICO STATE UNIV.

OREGON STATE UNIV.
Daryl Cooper

Department of Mathematics

University of California

Santa Barbara, CA 93106-3080 cooper@math.ucsb.edu

Jiang-Hua Lu

Department of Mathematics

The University of Hong Kong

Pokfulam Rd., Hong Kong

jhlu@maths.hku.hk

$$
\text { Jie Qing }
$$

Department of Mathematics

University of California

Santa Cruz, CA 95064

qing@ cats.ucsc.edu

\author{
UNIV. OF CALIF., SANTA CRUZ \\ UNIV. OF MONTANA \\ UNIV. OF OREGON \\ UNIV. OF SOUTHERN CALIFORNIA \\ UNIV. OF UTAH \\ UNIV. OF WASHINGTON \\ WASHINGTON STATE UNIVERSITY
}

These supporting institutions contribute to the cost of publication of this Journal, but they are not owners or publishers and have no responsibility for its contents or policies.

See inside back cover or msp.org/pjm for submission instructions.

The subscription price for 2016 is US $\$ 440 /$ year for the electronic version, and \$600/year for print and electronic.

Subscriptions, requests for back issues and changes of subscriber address should be sent to Pacific Journal of Mathematics, P.O. Box 4163, Berkeley, CA 94704-0163, U.S.A. The Pacific Journal of Mathematics is indexed by Mathematical Reviews, Zentralblatt MATH, PASCAL CNRS Index, Referativnyi Zhurnal, Current Mathematical Publications and Web of Knowledge (Science Citation Index).

The Pacific Journal of Mathematics (ISSN 0030-8730) at the University of California, c/o Department of Mathematics, 798 Evans Hall \#3840, Berkeley, CA 94720-3840, is published twelve times a year. Periodical rate postage paid at Berkeley, CA 94704, and additional mailing offices. POSTMASTER: send address changes to Pacific Journal of Mathematics, P.O. Box 4163, Berkeley, CA 94704-0163.

PJM peer review and production are managed by EditFLOW ${ }^{\circledR}$ from Mathematical Sciences Publishers.

PUBLISHED BY

\section{I. mathematical sciences publishers}

nonprofit scientific publishing

http://msp.org/

(C) 2016 Mathematical Sciences Publishers 


\section{PACIFIC JOURNAL OF MATHEMATICS}

Volume $285 \quad$ No. $2 \quad$ December 2016

The $\mathrm{SU}(N)$ Casson-Lin invariants for links

HANS U. BODEN and ERIC HARPER

The SU(2) Casson-Lin invariant of the Hopf link

HANS U. BODEN and CHRISTOPHER M. HERALD

Commensurations and metric properties of Houghton's groups

José BuRILlo, SEAn Cleary, ARMANDo MaRTino and ClaAs

E. RÖVER

Conformal holonomy equals ambient holonomy

ANDREAS ČAP, A. RoD GOVER, C. RoBIN GRAHAM and

MATTHIAS HAMMERL

Nonorientable Lagrangian cobordisms between Legendrian knots

ORSOLA CAPOVILLA-SEARLE and LISA TRAYNOR

A strong multiplicity one theorem for $\mathrm{SL}_{2}$

JINGSONG CHAI and QING ZHANG

The Yamabe problem on noncompact CR manifolds

PAK TUNG Ho and SeONGTAG KIM

Isometry types of frame bundles

WOUTER VAN LIMBEEK

Bundles of spectra and algebraic K-theory

JOHN A. LIND

Hidden symmetries and commensurability of 2-bridge link complements

CHRISTIAN MiLlichaP and WILLIAM WORDEN

On seaweed subalgebras and meander graphs in type $C$

DMITRI I. PANYUSHEV and OKSANA S. YAKIMOVA

The genus filtration in the smooth concordance group 